\title{
Bilateral Mastectomy for Breast Cancer Prevention
}

\author{
Leah Whitman, Kaitlyn Champion, Casey Clark and Adegoke Adeniji* \\ South University, School of Pharmacy, USA
}

Submission: January 09, 2018; Published: February 09, 2018

*Corresponding author: Adegoke Adeniji, School of Pharmacy, South University USA, Tel: 9122018157; Email: aadeniji@southuniversity.edu

\begin{abstract}
Breast cancer continues to be a major public health concern and cause of female mortality despite significant advancements in prevention methods. Surprisingly, regardless of the abundance of evidence supporting other methods of prevention, twice as many women are choosing bilateral mastectomy. Several trials have investigated the efficacy of removing healthy breast tissue to prevent cancer development and recurrence, yet such procedures remain controversial. Breast cancer recurrence, disease-free survival, cost-effectiveness, and quality of life postsurgery are critical components of a patient's decision to undergo prophylactic bilateral mastectomy. Selection of an appropriate prevention method requires an accurate and complete understanding of each available option. Here we review the current evidence concerning the use of bilateral mastectomy for breast cancer prevention, and compare the choice of undergoing the procedure to other less invasive prevention methods such as chemoprevention, which remains the gold standard for breast cancer prevention.
\end{abstract}

Keywords: Selective estrogen receptor modulators; BRCA1; BRCA2; Triple negative breast cancer, contralateral prophylactic mastectomy; Aromatase inhibitors

\section{Introduction}

\section{Epidemiology}

Breast cancer continues to be a major cause of female mortality despite significant medical advances and increased therapeutic options. There are over 3 million women with breast cancer in the United States, with an additional 300,000 projected to be diagnosed with the disease in 2016. Historically, breast cancer incidence and prevalence trended upward for decades, partly due to advances in diagnostic technology. In the 1980 s and 1990s, the incidence of breast cancer grew significantly after the development of mammography screenings. However, the incidence rates steadied in most subgroups of women by the early 2000s. This stabilization is attributed to increased breast cancer awareness paired with the ability of doctors to discover less developed and thus more manageable breast malignancies through improved screening techniques.

Risk factors for breast cancer include age, heredity, and race. The risk for breast cancer increases with age, regardless of race, with the median age at time of diagnosis being 61 years old. [1] Breast cancer is more common in Caucasian women followed by African American women. However, mortality due to breast cancer is higher in African American women. The overall 5-year relative survival rate for localized, non-invasive breast cancer in all races is $99 \%$. This survival rate declines as the cancer metastasizes. The 5-year survival rate for African American and comparable white women with invasive breast cancer is $17 \%$ and $27 \%$, respectively. [1].
Increase in breast cancer awareness and the findings of the Women's Health Initiative impacted mortality from the disease in the early 21st century. The Women's Health Initiative was a randomized placebo-controlled trial aimed at reducing heart disease, breast cancer and colorectal cancer in post-menopausal women that were treated with estrogen and progesterone. Complications in the treatment group led to the early termination of the trial. Notably, researchers saw an increase in breast cancer and cardiac complications in the treatment group relative to the placebo group. The reported complications consisted of heart disease, stroke, pulmonary embolism, and invasive breast cancer. [2,3] The findings from this trial sparked an interest in breast cancer education and the development of efficacious prevention and treatment methods.

\section{Classification of Breast Cancer}

There are two main classification of breast cancer as differentiated by location: in situ carcinoma and invasive carcinoma. In situ carcinoma is confined to a location usually within a duct or lobule in the breast. In contrast, invasive carcinoma spreads beyond the duct or lobule into the breast tissue and has the ability to spread to other areas of the body. In situ carcinoma is further classified as either ductal carcinoma in situ (DCIS) or lobular carcinoma in situ (LCIS). DCIS, also known as intraductal carcinoma, is an abnormal, often clinically detectable growth of cells confined to the breast duct without invasion of the surrounding tissue. DCIS is rare in women less 
than 30 years of age and is generally diagnosed around the time of menopause [4]. LCIS is an abnormal growth of cells in the secretory glands (lobules) of the breast and is classified as either "classic" or "pleomorphic." Classic lobular carcinoma involves less than four lobular ductal units while pleomorphic lobular carcinoma represents a more aggressive variant and is more likely to advance to invasive carcinoma. Unlike DCIS, lobular tumors are often not physically detected and are typically a secondary finding during a breast examination [5]. Both types of in situ carcinoma increase the risk for invasive carcinoma [6].

Invasive breast cancer is designated once the disease spreads beyond the epithelial tissue. Severity is then staged using the TNM system. Staging criteria is based on the size of the tumor $(\mathrm{T})$, involvement of lymph node $(\mathrm{N})$ in tumor progression and metastatic status (M). Stage 0 refers to non-invasive cancers such as DCIS and lobular carcinoma. Stages 1-4 are invasive cancers with stage 4 being the most advanced disease. Patients with stage 4 breast cancer have metastatic tumors and thus have the least favorable prognosis.

\section{Breast Cancer Subtypes}

The molecular characteristics of a breast tumor usually inform the choice of the most effective treatment. There are four molecular subtypes: Luminal A, Luminal B, Triple Negative, and HER-2 enriched (Table 1). These subtypes are defined by the presence (HR+) or absence (HR-) of hormone receptors (HR) as well as excessive (HER2+) or normal (HER2-) levels of human epidermal growth factor receptor 2 (HER2). Hormone receptors may be activated by estrogen, progesterone, or both. Luminal $\mathrm{A}$ is distinguished as hormone receptor positive and HER2 negative (HR+/HER2-) [7]. This molecular subtype tends to be slowing growing and less aggressive. Luminal B (HR+/HER2+), also known as triple positive, tends to be more aggressive than luminal A [8]. Triple negative (HR-/HER2-) breast cancer exists when cancer cells are negative for both estrogen receptors (ER) and progesterone receptors (PR) and do not have excess HER2. This subtype tends to grow and spread rapidly. HER2-enriched (HR-/HER2+) is also an aggressive form of breast cancer [9].

Table 1: Molecular Subtypes of Breast Cancer.

\begin{tabular}{|c|c|c|c|c|}
\hline & Luminal A & Luminal B & $\begin{array}{c}\text { HER2 } \\
\text { Enriched }\end{array}$ & $\begin{array}{c}\text { Triple } \\
\text { Negative }\end{array}$ \\
\hline HR & + & + & - & - \\
\hline HER2 & - & + & + & - \\
\hline Prognosis & $\uparrow$ & $\uparrow$ & $\downarrow$ & $\downarrow \downarrow$ \\
\hline
\end{tabular}

Patients with triple negative and HER2-enriched breast cancer have a poor prognosis and more limited treatment options than those with Luminal A or B carcinoma. The presence of a HR protein suggests a favorable prognosis when treated with hormone therapy. Depleting estrogen and/or progesterone or inhibiting their action suppresses HR activity and slows tumor progression. However, this anti-hormone approach provides little to no success for diseases lacking the hormone receptor. Excessive HER2 protein, such as in HER2 enriched patients, indicates a disease that will respond to tyrosine kinase inhibitors that specifically target HER2. Although HER2 enriched breast cancer still carries a poor prognosis, advancements in HER2 targeted therapies are promising [10,11]. Unlike HER2 and HR positive tumors, there are no targeted therapies against triple negative breast cancers with chemotherapy being the only choice (Table 1).

In addition to molecular subtypes, genetic mutations can influence the incidence and prognosis of breast cancer. The most clinically meaningful genetic mutations with regards to breast cancer to date are BRCA1 and BRCA2. These are considered susceptibility genes because these mutations alone do not cause patients to develop the disease. Rather, carcinoma in these patients results from a combination of genetic and epigenetic factors, such as exposure to carcinogens. While genetic testing to screen for BRCA mutations is available, its role in clinical decision making remains controversial. Patients with either BRCA1 or BRCA2 gene mutations have a significantly higher risk of developing breast cancer, 57\%-65\% and 45\%-55\%, respectively [12]. Despite the uncertainty behind the medical applicability of genetic testing, many patients with BRCA1/2 gene mutations consider prophylactic surgery a key preventive modality.

\section{Risk Factors}

In order to improve mortality associated with breast cancer, it is imperative to understand the risks associated with its development. To assess the risk of developing breast cancer from benign or non-cancerous breast masses, lesions may be classified into one of three categories. Non-proliferative lesions represent the lowest chance of developing cancer. Masses in this category cause breast tissue overgrowth but do not always increase the risk of breast cancer. Examples of conditions associated with non-proliferative lesions include simple cysts, adenosis, fat necrosis, and other benign tumors. Proliferative lesions without atypia (structural abnormality), however, cause an increase in tissue development in the ducts and lobules therefore increasing the risk of breast cancer. Examples of proliferative lesions include ductal hyperplasia, fibroadenoma, and several papillomas. Lastly, proliferative lesions with atypia cause an excessive growth of tissue in the ducts and lobules. This is distinguished from conditions without atypia by the presence of abnormal structures capable of differentiating into cancerous tissue. Such conditions include atypical ductal hyperplasia and atypical lobular hyperplasia.

Multiple risk factors have been implicated in the development of breast cancer. Non-modifiable risk factors include gender, age, race and ethnicity, inherited genes, family or personal history of breast cancer, breast tissue density, and radiation exposure. Of these, the principal risk factors are female gender and age. Because exposure to estrogen and progesterone is significantly greater in females, women are 100 times more likely to develop 


\section{Global Journal of Pharmacy \& Pharmaceutical Sciences}

breast cancer than men [13]. Furthermore, the greater life-time exposure to estrogen and progesterone in women appears to contribute to the development of breast cancer. This is supported by the increased incidence of breast cancer in post-menopausal women (especially those who enter menopause after age 55) and women who begin menstruating before age twelve. Also, most invasive breast cancers are found in postmenopausal women over the age of 55 [14]. In addition to age and sex, women with a family history of breast cancer, particularly a firstdegree relative, are at a higher risk of breast cancer development [15]. Studies have shown that women with a personal history of breast cancer in one breast are at an increased risk of developing cancer in the second breast [15]. This type of breast cancer is referred to as contralateral breast cancer, and indicates a second primary tumor. Primary breast cancer risk is also increased by inherited genes responsible for yielding dense breast tissue. Women with dense breasts (more glandular and fibrous tissue than fatty tissue) are two times more likely to develop breast cancer. Furthermore, mammograms are less accurate with dense breast tissue, which may result in false negative screenings and delayed treatment [16]. Radiation has also been linked to breast cancer development, though its impact is likely dependent on age. Chest X-rays in adolescents causes increased risk, especially if received during puberty/breast development. However, after the age of 40 , radiation does not appear to affect the risk of breast cancer. Interestingly, concurrent use of chemotherapy and radiation reduces the risk of developing breast cancer, possibly due to a chemotherapy-induced reduction in ovarian hormones [17].

Modifiable risk factors for breast cancer include alcohol consumption, obesity and physical inactivity, number of children, birth control use, and post-menopausal hormone therapy. Alcohol consumption is directly correlated with an increased risk. Women who consume 2 to 5 drinks per day are at 1.5 time's greater risk than those who consume one drink per day [18]. Obesity is a more complex risk factor than alcohol consumption. After menopause, fat tissue begins to produce estrogen to compensate for loss of optimally functioning ovaries. Increased adiposity in obese postmenopausal women causes increased estrogen levels, which increases the risk of breast cancer. This risk varies depending on various factors such as age of weight gain and location of the excess fat. Obese patients also have increased insulin levels, which has been linked to breast cancer [19].

Another modifiable risk factor is parity. A reduced risk has been seen in women who have multiple pregnancies and who give birth at a younger age. Furthermore, oral contraceptive therapy slightly increases risk, possibly due to increased life-time hormone exposure. Notably, after ten years, the risk of breast cancer in women who stopped using oral contraceptives was comparable to those who had never used oral contraceptives. Similarly, the risk of breast cancer development with injectable contraceptives returned to baseline 5 years after discontinuation
[20]. Other reversible risk factors include hormone replacement therapy (HRT). HRT is indicated for relief of menopause symptoms and as adjunctive therapy for prevention of osteoporosis in post-menopausal women. Estrogen therapy alone has shown no increase in risk when used short-term. However, some studies have shown an increase in breast and ovarian cancer after 10 years of estrogen therapy alone. Combined hormone replacement therapy consists of progesterone and estrogen and has also been shown to increase the risk of breast cancer [21]. As with other hormone therapies, the risk of breast cancer usually drops to that of the general population after discontinuation of hormone replacement therapy [22].

The decision to undergo preventative therapy for breast cancer typically depends on individual patient risk. The National Cancer Institute (NCI) Breast Cancer Risk Assessment Tool is often used to approximate the risk of developing invasive breast cancer within the next 5 years and for a lifetime (max 90 years of age). The factors used to calculate risk are listed in Table 2 . Women with a 5-year risk score of 1.67 percent or higher are considered at "high risk". This tool was adapted from the "Gail Model" which was the first valid risk assessment developed for Caucasian women. The newer model adjusts for race/ethnicity to include African American and Pacific Islanders. The NCI Breast Cancer Risk Assessment Tool is the most commonly used tool used to determine individual breast cancer risk, however, limitations exist. Scoring was developed exclusively for invasive breast cancer and the determining risk factors are not all-inclusive [23]. There are additional surveys that are more appropriate for women with certain medical conditions. For example, if a woman has the BRCA1/2 gene mutation, the BOADICEA model would be the most appropriate tool. In addition, if a woman has a history of LCIS, the IBIS Breast Cancer Risk Evaluation tool would provide a more accurate risk assessment for invasive breast cancer.

Table 2: Factors Used in NCl Risk Assessment Tool.

\begin{tabular}{|c|}
\hline Age ( $\geq 35$ years old), \\
\hline History of DCIS or LCIS \\
\hline BRCA1/2 mutation \\
\hline Age of first menstrual cycle \\
\hline Age at time of first birth \\
\hline Family history of breast cancer \\
\hline Number of breast biopsies \\
\hline Presence of atypical hyperplasia \\
\hline Race/ethnicity \\
\hline
\end{tabular}

\section{Mastectomy}

Regardless of the ability to control exposure to certain risk factors, many women remain at high risk for developing breast cancer. For some of these patients, the choice of a breast cancer prevention method can be a life-or-death decision. Over the last decade, prophylactic bilateral mastectomy has become a common method for breast cancer prevention, as it provides 
a reliable modality for limiting breast cancer development. A unilateral mastectomy, removal of one breast, is also used but this procedure does not lower the risk of cancer in the remaining breast. Several mastectomy subtypes with varying degrees of tissue removal have been defined including total (simple) mastectomy, radical mastectomy, modified radical mastectomy, nipple-sparing mastectomy, and partial mastectomy. The most commonly performed procedure is the total mastectomy which involves complete removal of the breast tissue in one or both breasts. The underlying muscle and lymph nodes remain intact with this type of mastectomy unless a lymph node exists within the breast tissue. This is the standard procedure for prophylaxis and women with large areas of DCIS. In contrast, a radical mastectomy is the most extensive surgery. It involves complete removal of the breast tissue, eradication of the lymph nodes located adjacent to the breast, and extraction of the underlying muscle (pectoralis major). Radical mastectomies are usually reserved for patients with cancer that has metastasized to the pectoralis major. A modified-radical mastectomy removes all breast tissue and axillary lymph nodes while the pectoralis major remains untouched. The modified radical mastectomy has largely replaced the radical mastectomy because similar therapeutic outcomes were observed with both procedures. A nipple-sparing mastectomy is a variant of total mastectomy that preserves the nipple/areola and breast skin. Lastly, a partial mastectomy, also called breast conservation surgery, removes only the tumor and surrounding tissue. A lumpectomy is similar to a partial mastectomy, but the amount of surrounding tissue removed is greater in partial mastectomy. Although tissue sparing techniques are attractive options for many patients, some women may not be candidates for partial removal [24].

\section{Current trends in prophylactic bilateral mastectomy}

Prophylactic bilateral mastectomy is an approved treatment for breast cancer risk reduction in women and awareness of the procedure has grown steadily in recent years. This heightened awareness, greater access to genetic testing, and improvements in reconstructive surgery options have made bilateral mastectomy an increasingly popular choice among women at risk of developing breast cancer. According to a retrospective cohort study examining trends in mastectomy rates from 1998-2011 in America, six times as many patients are choosing bilateral breast removal relative to the earlier time point (Figure 1). The procedure was more likely to be chosen over breast conservation surgery by women with advanced/invasive disease, no insurance; government funded insurance and low education level [25]. As the popularity of this procedure raises, evidence supporting its efficacy, safety, and utility compared to other available options is critical to appropriately educate patients considering mastectomy for breast cancer prevention.

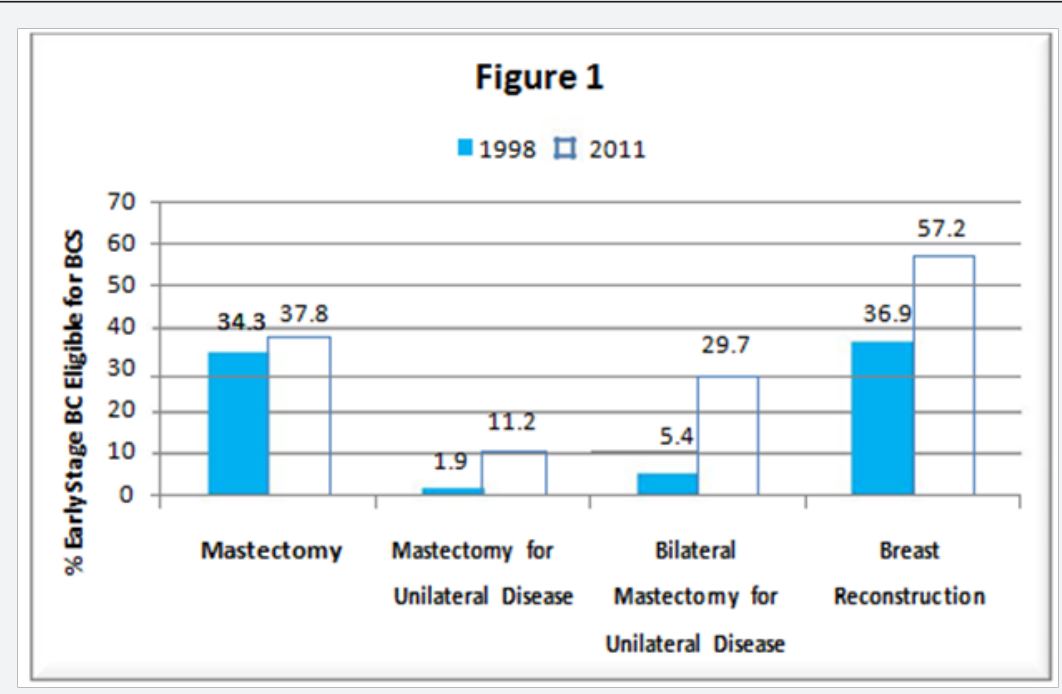

Figure 1: Trends in US women with early stage (BC) breast cancer choosing mastectomy over breast conservation surgery (BCS) for breast cancer prevention from 1998-2011(25).

\section{Efficacy of bilateral mastectomy in BRCA positive patients}

Various studies have demonstrated the efficacy of bilateral mastectomy for the prevention of breast cancer in high risk patients. The 2004 PROSE study, a prospective-retrospective multicenter study including European and North American women, found that prophylactic bilateral mastectomy significantly reduces the risk of breast cancer in BRCA1/2 mutation carriers [26]. Researchers used incidence density sampling to determine the benefit of bilateral mastectomy in women with confirmed BRCA1 or BRCA2 mutations. The test group included BRCA positive women with no prior history of breast cancer who underwent prophylactic bilateral mastectomy. For each woman in the test group, at least one patient of similar age, BRCA variance, and geographical location was assigned to the control group if she had no history of breast cancer or bilateral mastectomy on the date of the test subject's surgery. The researchers performed four analyses: all cases and controls, cases and controls with no prior oophorectomy, all prospective 
cases and controls, and all prospective cases and controls with no prior oophorectomy. In the largest analysis, breast cancer was diagnosed in $48.7 \%$ of the 378 controls while only two cases were detected in the test group after 6.7 and 5.5 years, respectively. Of note, the two cases of breast cancer in the test group both opted for a nipple sparing mastectomy, which leaves behind a considerable amount of susceptible tissue and thus is not the preferred method for surgical prevention. Of the prospectiveonly analyses, no test subjects were diagnosed with breast cancer after approximately 3 years of follow-up. The respective control group was found to have a significantly higher incidence of breast cancer though longer follow-up times may have yielded less dramatic results. Nonetheless, the findings from the PROSE study indicated a $90 \%$ risk reduction in the test group, making it the first study to estimate the magnitude of benefit derived from preventative bilateral mastectomy. The general findings were consistent with a smaller study conducted by Heijboer et al. [27] which also evaluated the risk of developing breast cancer after prophylactic bilateral mastectomy in 139 women with a BRCA mutation. The study had a shorter follow-up period than the PROSE study and was completed at a single institution. The results showed no cases of breast cancer in the 76 women who had a bilateral mastectomy compared to 8 cases in the 63 women who were just actively monitored. The similar outcomes of these two studies are especially impactful in determining riskmanagement strategies for patients who are positive for BRCA mutations. However, results may not be generalizable to patients without those mutations.

\section{Efficacy of bilateral mastectomy in high risk, BRCA negative patients}

Evidence for the efficacy of bilateral mastectomy exists in patients with confirmed genetic predispositions, but studies have also shown benefit in other high risk groups. A recent study showed that BRCA negative, early breast cancer patients who also have a family history of breast cancer are at a higher risk for contralateral breast cancer [28]. The risk was higher in patients with first degree family history as opposed to patients with a second degree relative. Furthermore, those patients who underwent total bilateral mastectomy for the prevention of contralateral breast cancer had a slightly greater 20-year overall survival and disease-free period. Overall 20-year absolute disease free survival rate following mastectomy was highest $(12.59 \%)$ in breast cancer patients with a first degree relative with bilateral breast cancer and lowest $(0.93 \%)$ in patients with no family history.

This considerable disparity in rate reduction indicates the existence of other important genetic factors outside of BRCA mutations. The study also found that age was an important factor when considering the survival benefit of the operation for early stage breast cancer patients. Women under the age of 40 , regardless of family history, with either stage I or II breast cancer saw the greatest benefit after undergoing bilateral mastectomy even though they had unilateral breast cancer, a procedure referred to as contralateral prophylactic mastectomy (CPM). Contralateral prophylactic mastectomy was especially unfavorable after 60 years old, and the procedure was less beneficial as patient's age and stage of disease development increased.

Though many studies have shown an impressive reduction in breast cancer recurrence, a meta-analysis of CPM after unilateral breast cancer revealed that the operation had no impact on absolute overall survival in patients with certain high risk factors [29]. In the study, stratification of subgroups by family/genetic risk showed improved relative risk but no absolute risk reduction between overall survival and CPM in patients with high genetic risk factors or family history. However, the relative and absolute risk of contralateral breast cancer incidence and distant recurrence was notably decreased in these patients. The study notes that the risk of contralateral recurrence of primary breast cancer is minute in the general population, and overall survival improvements at large could be attributed to confounding variables other than the procedure. Without the surgery, an estimated $10 \%$ of unilateral breast cancer patients without a BRCA mutation are projected to develop a contralateral primary tumor. However, under the same circumstances almost half of BRCA positive patients may have a recurrence in the opposite breast. This would explain the stronger impact the procedure has on genetically predisposed patients and supports the recommendation that radical bilateral mastectomy be reserved for unilateral breast cancer patients with disease-susceptible mutations or a strong family history. The results of another study spur skepticism in the necessity of bilateral mastectomy regardless of genetic predisposition.

In 2002, the National Surgical Breast and Bowel Project (NSBBP) published the results of a 20 year follow up of a randomized clinical trial designed to compare survival rates of 1,851 women after total mastectomy, lumpectomy, or lumpectomy with irradiation for treatment of breast cancer [30]. The findings revealed no difference in overall survival, diseasefree survival, or distant disease-free survival among the three treatment groups. This lack of survival benefit is consistent with the trial's 10 year findings as well as results from a 1995 study by the Early Breast Cancer Trialists' Collaborative Group (EBCTCG) which determined no statistical difference in overall survival between patients undergoing mastectomy vs breast conserving surgery after 10 years [30,31]. Table 1 summarizes the findings of various trials exploring the efficacy of mastectomy for breast cancer prevention. No data was reported on patient satisfaction or cost-effectiveness. As multiple studies have reported no significant survival differences between prophylactic bilateral mastectomy and other various prevention methods, future studies should focus on differences in patient satisfaction and quality of life, as these factors are likely important in shared decision making (Table 3). 


\section{Global Journal of Pharmacy \& Pharmaceutical Sciences}

Table 3: The cost of total mastectomy with chemoprevention, the monthly cost for various agents used to reduce the risk of breast cancer.

\begin{tabular}{|c|c|c|c|}
\hline Study & Population & Results $^{* * *}$ & Median Follow Up \\
\hline EBCTCG [31] & Mastectomy vs BCS & No difference in OS & 10 years \\
\hline Hartmann et al. [32] & Family history $\mathrm{n}=639$ & $\downarrow$ BC Incidence: $~ 90 \% \downarrow$ BC Death Incidence: $~ 90 \%$ & 14 years \\
\hline PROSE Study Group [26] & BRCA1 \& BRCA2 n=289 & $\downarrow$ BC Risk: $90 \%$ & $\begin{array}{l}5.7 \text { years (Cases) } 6.7 \\
\text { years (Controls) }\end{array}$ \\
\hline *Meijers-Heijboer et al. [27] & BRCA1 \& BRCA2 n=139 & $\downarrow \mathrm{BC}$ Incidence: HR, 0 & 3 years \\
\hline NSBBP [30] & $\begin{array}{l}\text { Mastectomy vs Lumpectomy } \\
\mathrm{n}=1,851\end{array}$ & $\begin{array}{l}\text { No difference in overall survival, disease-free survival, or } \\
\text { distant disease-free survival }\end{array}$ & 20 years \\
\hline Bedrosian [33] & $\mathrm{CPM}^{* *} \mathrm{n}=107,106$ & $\begin{array}{l}\uparrow \text { Disease-Free Survival HR: } 0.84(\text { age }<50) \text { HR: } 0.79 \text { (age } \\
\text { 50-59) No difference }(\text { age }>60)\end{array}$ & 47 months \\
\hline $\begin{array}{c}\text { Fayanju et al. [29] } \\
\text { (Review \& Meta-Analysis) }\end{array}$ & CPM for UBC $¥ \mathrm{n}=14$ studies & $\begin{array}{c}\text { Full Analysis: } \uparrow \text { Overall Survival: } 9 \% \downarrow \text { BC Mortality: } 31 \% \\
\text { Stratified Meta-Analysis: } \downarrow \text { Metachronous Contralateral } \\
\text { BC**** incidence only: }>90 \%\end{array}$ & \\
\hline
\end{tabular}

Key:

${ }^{*}$ Prospective study

${ }^{* *}$ CPM-Contralateral Prophylactic Mastectomy (Only total and modified radial mastectomies included) $¥$ Unilateral Breast Cancer.

***Findings - All findings listed were statistically significant.

${ }^{* * * *}$ A second malignancy diagnosed $>6$ months after primary tumour, located in a different area.

\section{Risk of recurrence and survival outcomes}

Although prophylactic bilateral mastectomy significantly reduces the chance of developing breast cancer in certain patient populations, prevention is never guaranteed. This is because complete removal of susceptible glandular tissue is unattainable even in radical mastectomies, due to deep tissue intermixtures within the chest wall and axillary tail. [32-34]. As early as 1940, studies have shown the presence of residual glandular tissue after a total mastectomy [35]. In 2013, Griepsma et al. [36] found glandular breast tissue at the resection plane in over $75 \%$ of standard (mostly total) mastectomies. Based on his data, the risk of residual tissue resulting in breast cancer recurrence postsurgery after a total mastectomy may be as high as 1 in every 140 women. In previously diagnosed breast cancer patients, the risk of recurrence may be increased by young age, large tumors, nodal involvement, or additional unfavorable genetic mutations [34]. Interestingly, although studies show significant reduction in tumor recurrence after bilateral mastectomy in patients with BRCA1/2 mutations, no impact has been observed on overall mortality in patients with BRCA1/2 mutations. [27,29]. In addition to breast cancer recurrence, patients, especially those with BRCA mutations may still be at an increased risk for ovarian cancer after a bilateral mastectomy. According to Domcheck et al. [37] a woman's lifetime risk of developing ovarian cancer was $36 \%-63 \%$ for BRCA1 mutation carriers and $10 \%-27 \%$ for BRCA2 mutation carriers.

\section{Patient Satisfaction}

The risk of infection, pain, and bleeding abnormalities exists after prophylactic mastectomy; however, common complications of the procedure are relatively mild. Aside from the physical toll, the experience may also have a substantial impact on a woman's mental health. After surgery, women often experience psychological side effects such as depression due to reduced body image satisfaction and loss of femininity. A swedish study found that satisfaction among women that had undergone prophylactic mastectomies followed by immediate breast reconstruction ranged from $60-74 \%$ [38]. Although the sample size of 24 was relatively small, the majority of patients were in fact satisfied with the breast reconstructive surgery overall. The most common complaint concerned the nipple-areola complex, and many subjects chose to undergo additional surgery. Preserving the nipple-areola complex (NAC) may improve self-image and sensation. Unfortunately, nipple-sparing mastectomy has been shown to increase the risk of breast cancer recurrence, though it may not effect overall survival [34]. A recent retrospective study of nearly 160,000 women over a 17-year period found no significant difference in survival between patients receiving nipple-sparing mastectomy vs. non-nipple sparing mastectomy in California [39]. This is consistent with previous studies that found no improvement in survival among patients using various surgical breast cancer prevention methods regardless of genetic predisposition.

Even though many normal breast functions are lost even after nipple sparing mastectomy, the lack of any long term survival benefit with non-nipple sparing procedures may influence a patient's decision to preserve the NAC and possibly improve psychological morbidity. Furthermore, advanced reconstructive surgery accessibility may offer solutions to poor body image without increasing future risk of recurrence. As of 1998, the Women's Health and Cancer Rights Act mandates the coverage of reconstructive surgery following mastectomy if the mastectomy was covered by the patient's insurance plan. Under these circumstances, coverage applies to any available reconstructive method as deemed appropriate by the patient, including long term modifications. The law provides the patient with more control over the aesthetic outcome of the surgery, possibly improving the likelihood of post-operative satisfaction. Benjamin H.L. et al., found that women who underwent mastectomies with reconstructive surgery had similar quality of life outcomes 


\section{Global Journal of Pharmacy \& Pharmaceutical Sciences}

to patients choosing breast conservation surgery [40]. In this cross-sectional cohort study, BREAST-Q questionnaires were completed by women following breast conservation surgery, total mastectomy with or without reconstructive surgery, and women without breast cancer. The results showed that women who underwent a total mastectomy with reconstruction and women who underwent breast conservation surgery were statistically more 'satisfied with breast' as opposed to women who underwent a total mastectomy without reconstructive surgery. Similar results were seen in the sexual well-being' domain. While beneficial immediate reconstructive surgery has been associated with a higher incidence of surgical site infections, especially in diabetic patients [41,42]. Efforts should be made to ensure all patients understand the risks and benefits of less aggressive procedures and are aware of health insurance plan benefits in terms of reconstructive surgery. Options for post-mastectomy modifications, however, may be limited for patients without medical insurance coverage.

\section{Breast Cancer Chemoprevention}

Patients reluctant to remove breast tissue may consider pharmacological agents as an alternative prevention method. Only two drugs are currently approved by the US Food and Drug Administration (FDA) for chemoprevention of breast cancer: tamoxifen and raloxifene. These drugs are selective estrogen receptor modulators (SERMs) and are only used in high-risk patients. Researchers are also exploring the use of aromatase inhibitors, which are currently FDA approved for breast cancer treatment only. Aromatase inhibitors have shown promising results when used for breast cancer chemoprevention. Patients and physicians must examine the efficacy and safety of these agents when deciding if and when chemoprevention is a more appropriate option than surgical prevention [43].

\section{Selective Estrogen Receptor Modulators}

Selective estrogen receptor modulators (SERMs) target estrogen receptors in specific tissues. SERMs competitively inhibit estrogen receptor (ER) activation but may have agonistic estrogen effects in other areas of the body. The paradoxical effects of SERMs may result from activity at both $\alpha$ - and $\beta$-estrogen receptors which are thought to have contrasting functions based on their distribution patterns in various tissues. It may also be due to differential cellular expression of various coactivators or corepressors that can modulate the interaction of the ER with the ER dependent genes. Tamoxifen has anti-estrogenic effects in breast tissue and estrogenic effects on the endometrium and bone, whereas raloxifene has antiestrogenic effects in both breast and endometrial tissue, but estrogenic effects on the bone. While both agents are currently approved for breast cancer prevention, each has an overlapping but distinct mechanism of action and adverse effect profile.

Tamoxifen reduces the risk of estrogen receptor positive $(\mathrm{ER}+)$ invasive and noninvasive breast cancers by approximately $50 \%$ in populations with various risk factors and has no activity against estrogen receptor negative cancers [44]. The anticancer activity of tamoxifen is attributed to the reduction in the expression of estrogen dependent genes. In addition, tamoxifen also disrupts the cell cycle through actions in the nucleus. It represses the expression of tumor inducers such as insulin-like growth factor 1, and promotes expression of tumor suppressor genes, such as transforming growth factor beta (TGF-beta) and maspin. Maspin concentration is significantly higher in normal breast cells when compared to cancer cells, and its activity may be particularly important in reducing invasion and metastasis. Tamoxifen is a cytostatic agent as it halts the cell cycle progression [45].

Table 4: Cost of Breast Cancer Risk Reduction Medications.

\begin{tabular}{|c|c|c|c|c|}
\hline & \multicolumn{2}{|c|}{$\begin{array}{c}\text { Selective Estrogen } \\
\text { Receptor Modulators }\end{array}$} & \multicolumn{2}{c|}{ Aromatase Inhibitors* } \\
\hline Drug & Tamoxifen & Raloxifene & Anastrozole & Exemestane \\
\hline Dose $¥$ & $20 \mathrm{mg}$ & $60 \mathrm{mg}$ & $1 \mathrm{mg}$ & $25 \mathrm{mg}$ \\
\hline AWP $^{\wedge}$ & $\$ 113.64$ & $\$ 213.84$ & $\$ 572.84$ & $\$ 606.94$ \\
\hline
\end{tabular}

*Off-label indication

¥Recommended daily dose for 5 years.

^AWP: Average Wholesale Price; generic medication; 30-day supply.

As a result of tamoxifen's estrogenic activity in the endometrium, uterine/endometrial cancer may develop with use. The National Surgical Adjuvant Breast and Bowel Project P-1 Study (NSABP P-1), a large randomized, placebo-controlled trial concluded that patients using tamoxifen for breast cancer prevention were two and a half times more likely to develop endometrial cancer when compared to placebo [46]. The incidence was higher in women over 50 years old, however the benefits of tamoxifen therapy outweighs the risk. Other notable adverse effects of tamoxifen include hot flashes, bone marrow suppression, thromboembolic events, liver toxicity, and cataracts. Tamoxifen carries two black box warnings to highlight evidence showing increased risk of uterine or endometrial cancer and life-threatening thromboembolic events such as stroke and pulmonary emboli. It is also important to note that recent studies suggest CYP2D6 polymorphisms may impact treatment outcomes of tamoxifen therapy. Tamoxifen, a prodrug, is metabolized by CYP2D6 to a major active metabolite, endoxifen. Low levels of endoxifen have failed to reduce breast cancer recurrence whereas high concentrations were associated with a $26 \%$ decrease, according to a large retrospective study done by Women's Healthy Eating and Living (WHEL) [47]. The WHEL study also revealed a strong association between CYP2D6 variants and endoxifen levels. A previous study reported significant reductions in recurrence-free survival and endoxifen levels in patients with CYP2D6 polymorphisms being treated with tamoxifen [48]. However, despite evidence showing reduced efficacy in CYP2D6-poor metabolizer status, CYP2D6 genotyping prior to tamoxifen therapy has not been proven to be an effective outcome predictor, especially when compared to endoxifen blood levels [49]. Nonetheless, the Endocrinologic and 


\section{Global Journal of Pharmacy \& Pharmaceutical Sciences}

Metabolic Drugs FDA Advisory Committee recommend including a precaution for poor CYP2D6 metabolizers with tamoxifen prescriptions. Such patients may be at an increased risk for treatment failure and thus breast cancer recurrence (Table 4).

Raloxifene was FDA approval for breast cancer prevention in 2007. Raloxifene exhibits ER antagonist activity in both breast and endometrial tissue while maintaining estrogenic activity in bones. This altered estrogen receptor modulation drastically reduces the incidence of uterine and endometrial cancers when compared to tamoxifen while maintaining bone strength and breast cancer prevention. These characteristics were highlighted in the Multiple Outcomes for Raloxifene Evaluation (MORE) trial, a multi-center randomized placebo-controlled trial that assessed the ability of raloxifene to reduce the risk of in situ and invasive breast cancer in post-menopausal women with osteoporosis. The resulting data revealed a statistically significant $65 \%$ overall risk reduction and a 90\% risk reduction associated with ER+ cancers. [50].

The Study of Tamoxifen and Raloxifene (STAR) compared the efficacy of tamoxifen and raloxifene for breast cancer chemoprevention in postmenopausal women. While no difference in efficacy was observed with short term use, long term follow-up (7 years) of participants revealed tamoxifen to be superior with a $50 \%$ reduction in breast cancer compared to a $38 \%$ reduction with raloxifene. Expectedly, the difference in incidence of uterine cancer was statistically significant. The annual incidence rate of uterine cancer in the tamoxifen group was 2.25 per 1,000 patients in comparison to the raloxifene group with 1.23 per 1,000 patients. Based on these annual incidence rates, participants taking raloxifene were $38 \%$ less likely to develop uterine cancer, though the difference in cumulative 7-year incidence rates was not statistically significant. Additionally, the rates of uterine hyperplasia and total hysterectomy during the study period were reduced by $84 \%$ and $61 \%$, respectively. There was no difference in incidence of transient ischemic attacks, strokes, severe angina, myocardial infarction, pulmonary embolism, and DVT. Although differences in the risk of in situ carcinomas such as LCIS and DCIS were not statistically significant in the STAR trial, placebo-controlled studies have shown tamoxifen to be superior at preventing in situ carcinomas. In addition to the differing adverse effect profile of tamoxifen and raloxifene, they also show markedly different efficacy in premenopausal women. Raloxifene currently has little to no supportive evidence for use in premenopausal women, while tamoxifen is the only approved agent for breast cancer prevention in this population [51]. Both drugs carry a black box warnings for an increased risk of deep vein thrombosis and pulmonary embolism, and a risk of death due to stroke. Although raloxifene is a category $\mathrm{X}$ while tamoxifen is category $\mathrm{D}$, the use of both agents is discouraged in pregnancy. Raloxifene is also contraindicated in breast-feeding. Such characteristics limit the use of these agents for patients with a history of DVT, on concurrent warfarin therapy, currently pregnant and/or nursing, or planning to become pregnant.

\section{Aromatase Inhibitors}

Although aromatase inhibitors are FDA-approved for the treatment of ER+ breast cancer, medications in this class are often used off-label to reduce the risk of invasive breast cancer in postmenopausal women [52]. Aromatase catalyzes the rate limiting step in estrogen biosynthesis by converting androstenedione and testosterone to estrone and estradiol, respectively. Aromatase is over expressed in certain breast cancers, but is also found in all cells that produce estrogen such as those of the adrenal gland, gonads, prostate gland, adipose, skin, and brain [53]. Aromatase inhibition is particularly important for postmenopausal women as they depend on peripheral tissues as a primary source for estrogen. Because these agents are more active in extra-glandular sites, aromatase inhibitors are much less efficacious in women with functioning ovaries. Inhibition of aromatase suppresses proliferation of ER+ breast cancer cell by depleting the tumor's fuel source. Agents belonging to this class of medications are categorized by chemical structure as either steroidal (exemastane) or non-steroidal (letrozole, anastrazole). Anastrozole and exemastane have proven efficacy in breast cancer treatment and prevention, but the use of letrozole for breast cancer prevention is unsupported.

Exemestane is an irreversible, steroidal aromatase inhibitor which reduces plasma estrone, estradiol, and estrone sulfate levels. Its steroidal structure allows tight binding to the active site of aromatase to cause a near complete inhibition. The most commonly reported adverse effects include moderate hot flashes, fatigue, and depression as well as mild alopecia, anxiety, and insomnia. Severe bone fractures and visual impairment has also been reported [54]. To assess the use of exemestane for breast cancer prevention, a randomized double-blinded, placebocontrolled trial, Exemestane for Breast-Cancer Prevention in Postmenopausal Women Trial (also known as MAP.3), compared exemestane to a placebo over five years [52]. After 3 years, exemastane reduced the annual relative incidence in invasive breast cancer by $65 \%$. The results of MAP. 3 demonstrated the use of exemestane to be a viable alternative to that of tamoxifen and/or raloxifene for breast cancer prevention. No significant differences were found in reports of osteoporosis, cardiac events, or bone fractures when compared to placebo, suggesting a more favorable side effect profile than that of SERMs.

Anastrozole is a competitive, non-steroidal inhibitor of aromatase which only reduces serum estradiol, unlike exemestane. Although it is the gold standard for ER+ early breast cancer treatment, its use has not been FDA approved for breast cancer prevention. Nevertheless, results from the Arimidex (Anastrozole), Tamoxifen, Alone or in Combination (ATAC) trial support its use in clinical practice [55]. The trial compared the long term (5 year) efficacy of either anastrozole or tamoxifen in preventing post-lumpectomy breast cancer recurrence in postmenopausal women. Anastrozole was found to be equally effective in reducing the incidence of $\mathrm{ER}+$ breast cancer recurrence, and patients using anastrozole were $53 \%$ less 
likely to develop contralateral disease compared to tamoxifen. Furthermore, disease free survival (DFS) and time to recurrence were both prolonged with the use of anastrozole. Adverse effects such as gynecological abnormalities, vascular events, fractures, and arthralgia were likewise lower in the anastrozole group. Similar to exemestane, anastrozole may be a preferred therapy to tamoxifen due to a mild adverse effect profile. An additional trial that investigated the role of anastrozole in breast cancer prevention was completed by the International Breast Cancer Intervention Studies (IBIS-II Prevention trial) [56]. The double blinded, randomized placebo-controlled trial assessed the efficacy of anastrozole for breast cancer prevention in high-risk post-menopausal women when taken once daily for 5 years.

Anastrozole participants were significantly less likely to develop primary breast cancer compared to the general public (placebo). Unfortunately, researchers saw a significantly increased incidence of vasomotor and musculoskeletal adverse events including moderate arthralgia and joint stiffness. However, no significant difference in thromboembolic events, cerebrovascular events, or myocardial infarctions was seen between groups. Both groups displayed decreased bone mineral density (BMD) from baseline which poses a concern for women at an increased risk for osteoporosis. To assess the need for adjuvant bisphosphonate therapy, a BMD scan is recommended prior to initiation of all aromatase inhibitors.

The adverse effects profile of exemestane and anastrozole are different from those of SERMS and are also less severe. The most common adverse effects are related to menopausal symptoms which can be particularly troublesome for many patients. Exemestane appears to have less effect on the bone and vasculature while anastrozole displays less central nervous system issues. Unlike SERMs, aromatase inhibitors lack a black box warning. Though more evidence supports the use of tamoxifen and raloxifene for breast cancer risk reduction, aromatase inhibitors are generally utilized when tamoxifen or raloxifene are contraindicated (i.e. thromboembolic events). Currently, the duration of therapy is five years though there is a lack of supporting clinical data. According to the NCCN guidelines, there is no current evidence for use of aromatase inhibitors in BRCA1/2 carriers.

\section{Cost Comparison-Mastectomy, Surveillance, and Chemoprevention}

A cost-effective analysis focusing on direct medical costs for early-stage, node negative, unilateral breast cancer patients was conducted by Zendejas B etal. [57]. Two contralateral breastcancer strategies were compared: unilateral mastectomy followed by surveillance and contralateral prophylactic mastectomy (CPM). Cohorts were divided into age groups in intervals of five from 45 to 75 years of age. Each simulation consisted of 10,000 patients. Cost parameters were obtained from the Nationwide Inpatient Sample database and Medicare reimbursements, and costs were adjusted to 2007 rates. Age at time of treatment played an important role in cost-effectiveness. As age increased, the costeffectiveness of CPM decreased, becoming cost-ineffective at age 70. Lifetime cost (breast cancer related treatment until death) for women at the age of 45 undergoing a CPM was $\$ 1412$ higher than that of surveillance. The model demonstrated significantly positive results for CPM in BRCA-positive patients of all ages. For this patient population, results revealed both a decrease in cost and an increase in quality of life. While the findings from this trial seem to validate the cost-effectiveness of CPM in unilateral breast cancer patients, comparing estimates of the direct and indirect cost associated with each case would provide a more convincing declaration [57].

Deshmukh et al. [58] conducted a retrospective study for women treated for stage I to III breast cancer with or without CPM. Cost estimates were conducted using Medicare reimbursements for professional and technical costs over a period of 24 months after diagnosis. All costs were adjusted for 2010 rates. This study found that the average cost of CPM was $\$ 7,759$ higher than that of other surgical forms of breast cancer treatment, although there was a $\$ 21,100$ difference in favor of immediate CPM vs delayed. A large discrepancy exists in the cost estimates used by Zendejas \& Deshmukh $[57,58]$. Costs reported by Deshmukh et al [58]. for a 24-month time period was much greater than the life time cost reported by Zendejas et al.[57]. Prices projected in the more recent study, Deshmukh [58] could reflect inflation, data collection methods, and/or a more extensive consideration of patient cost. Deshmukh et al. [58] also incorporated costs for length of hospital stay, medications, and laboratory tests whereas the simulation designed by Zendejas et al. [57] did not account for such patient variation. Differing methods of data collection may have also led to conflicting price estimates. Deshmukh et al. [58] retrospectively assessed 904 patients from 1997 to 2009 then adjusted the total costs based on the value of the dollar in 2010. The more recent data is likely the most reliable for current considerations.

To compare the cost of total mastectomy with chemoprevention, the monthly cost for various agents used to reduce the risk of breast cancer can be found in Table 3. Prices are listed as average wholesale price for a 30-day supply. At a cost of $\$ 113.64$ a month, tamoxifen $20 \mathrm{mg}$ over a 5-year period would cost almost $\$ 7,000$. Alternatively, a 5 year exemestane regimen for breast cancer risk reduction would be approximately over $\$ 36,000[59,60]$. This price is comparable to the cost of CPM according to Zendajas et al. [57] Other considerations such as cost associated with prescription pick up, laboratory monitoring, and follow-up as well as morbidity associated with adverse effects must be considered for each patient. The most recent study to determine the cost-effectiveness of bilateral prophylactic mastectomy concluded that the procedure may only be appropriate for women with a lifetime breast cancer risk of at least $50 \%[61,62]$. The study used a Markov model to account for changes over time and assumed willingness-to-pay thresholds of $\$ 50,000$ and $\$ 100,000$ for women ages $30+$ gaining 
a 90\% risk reduction. According to these cost parameters, quality adjusted life years gained were only cost-effective in women with a $51 \%$ and $57 \%$ risk, respectively. Deciding on a breast cancer prevention method is a complicated endeavor that involves highly individualized dynamics. It is important to consider life-time or long-term in addition to short-term cost when communicating this information to patients, particularly those of low socioeconomic status. Given the evidence for the cost-effectiveness of breast cancer prevention methods, it is clear that more attention should be placed on educating patients.

\section{Conclusion}

Bilateral prophylactic mastectomy is an irreversible and relatively effective breast cancer prevention method for a subset of women. The procedure has been shown to substantially reduce the incidence of breast cancer in patients with a strong family history and/or documented high-risk genetic mutation(s). It also substantially reduces the risk of contralateral tumors and invasive diseases in certain patients with LCIS, DCIS and unilateral breast cancer. However, none of these studies reported an improvement in overall survival, disease-free survival, or distant disease-free survival after 10 and 20 years when compared to more conservative options. Nevertheless, mastectomy procedures have become an increasingly popular choice even in patients for which such drastic measures are not indicated. Interestingly, patients who considered their therapy to be directed by physician recommendation rather than self-guidance often chose breast conserving surgery over mastectomy. This observation illustrates the importance of patient education and shared decision making.

Bilateral mastectomy represents a viable choice for breast cancer prevention in BRCA+ patients or those $<40$ years of age with a personal or strong family history of invasive breast cancer. Chemoprevention methods coupled with increased surveillance have also shown significant efficacy, although medications are only effective at preventing ER+ breast cancers. This information may be helpful in determining insurance coverage, but more studies are needed to compare the costs of prophylactic mastectomy with breast conserving surgery, pharmacologic preventions, and active surveillance. This data would be useful in developing an algorithm for determining whether a patient is a suitable candidate for prophylactic mastectomy considering that patients may get similar benefit from other options. Lastly, it is essential that physicians, surgeons, pharmacist and other healthcare workers emphasize patient education to reduce anxiety and improve satisfaction with more conservative treatment approaches as research shows no survival benefit among the varying degrees of surgical prevention methods.

\section{References}

1. Howlader NNA, Krapcho M, Miller D, Bishop K, Altekruse SF, et al. (2016) SEER Cancer Statistics Review, National Cancer Institute. Bethesda, Maryland, pp. 1975-2013.
2. Rossouw JE, Anderson GL, Prentice RL, LaCroix AZ, Kooperberg Cet al. (2002) Risks and benefits of estrogen plus progestin in healthy postmenopausal women: principal results From the Women's Health Initiative randomized controlled trial. JAMA 288(3): 321-333.

3. Stefanick ML, Cochrane BB, Hsia J, Barad DH, Liu JH, et al. (2003) The Women's Health Initiative postmenopausal hormone trials: overview and baseline characteristics of participants. Ann Epidemiol 13(9 Suppl): S78-S86.

4. Allegra CJ, Aberle DR, Ganschow P, Hahn SM, Lee CN, et al. (2009) NIH state-of-the-science conference statement: diagnosis and management of ductal carcinoma in situ (DCIS). NIH Consens State Sci Statements 26(2): 1-27.

5. Simpson PT, Gale T, Fulford LG, Reis-Filho JS, Lakhani SR (2003) The diagnosis and management of pre-invasive breast disease: pathology of atypical lobular hyperplasia and lobular carcinoma in situ. Breast Cancer Res 5(5): 258-262.

6. Oppong BA, King TA (2011) Recommendations for women with lobular carcinoma in situ (LCIS). Oncology Williston Park 25(11): 1051-1058.

7. Anderson WF, Rosenberg PS, Prat A, Perou CM, Sherman ME (2014) How many etiological subtypes of breast cancer: two, three, four, or more? J Natl Cancer Inst 106(8): DOI: 10.1093/jnci/dju165.

8. Parise C, Caggiano V (2014) Breast Cancer Survival Defined by the ER/ PR/HER2 Subtypes and a Surrogate Classification according to Tumor Grade and Immunohistochemical Biomarkers. Journal of Cancer Epidemiology 2014: 1-11.

9. Blows FM, Driver KE, Schmidt MK, Broeks A, van Leeuwen FE, et al. (2010) Subtyping of breast cancer by immunohistochemistry to investigate a relationship between subtype and short and long term survival: a collaborative analysis of data for 10,159 cases from 12 studies PLOS medicine 7(5): e1000279.

10. Wolff AC, Hammond ME, Hicks DG, Dowsett M, McShane LM, et al. (2013) Recommendations for human epidermal growth factor receptor 2 testing in breast cancer: American Society of Clinical Oncology/College of American Pathologists clinical practice guideline update. J Clin Oncol 31(31): 3997-4013.

11. Schroeder RL, Stevens CL, Sridhar J (2014) Small molecule tyrosine kinase inhibitors of ErbB2/HER2/Neu in the treatment of aggressive breast cancer. Molecules 19(9): 15196-15212.

12. Antoniou A, Pharoah PD, Narod S, Risch HA, Eyfjord JE, et al. (2003) Average risks of breast and ovarian cancer associated with BRCA1 or BRCA2 mutations detected in case Series unselected for family history: a combined analysis of 22 studies. Am J Hum Genet 72(5): 1117-1130.

13. (2015) Surveillance, Epidemiology and End Results (SEER) Program (www. seer.cancer.gov) SEER*Stat Database: NAACCR Incidence - CiNA Analytic File, 1995-2012, for NHIAv2 Origin, Custom File With County, ACS Facts and Figures Projection Project, North American Association of Central Cancer Registries, USA.

14. Collaborative Group on Hormonal Factors in Breast C (2012) Menarche, menopause, and breast cancer risk: individual participant meta-analysis, including 118964 women with breast cancer from 117 epidemiological studies. Lancet Oncol 13(11): 1141-1151.

15. Collaborative Group on Hormonal Factors in Breast C (2001) Familial breast cancer: collaborative reanalysis of individual data from 52 epidemiological studies including 58,209 women with breast cancer and 101,986 women without the disease. Lancet 358(9291): 13891399.

16. Bertrand KA, Scott CG, Tamimi RM, Jensen MR, Pankratz VS, et al. (2015) Dense and nondense mammographic area and risk of breast cancer by age and tumor characteristics. Cancer Epidemiol Biomarkers Prev 24(5): 798-809. 


\section{Global Journal of Pharmacy \& Pharmaceutical Sciences}

17. Travis LB, Hill DA, Dores GM, Gospodarowicz M, van Leeuwen FE, et al. (2003) Breast cancer following radiotherapy and chemotherapy among young women with Hodgkin disease. JAMA 290(4): 465-475.

18. Hamajima N, Hirose K, Tajima K, Rohan T, Calle EE, et al. (2002) Alcohol, tobacco and breast cancer--collaborative reanalysis of individual data from 53 epidemiological studies, including 58,515 women with breast cancer and 95,067 women without the disease. Br J Cancer 87(11): 1234-1245.

19. Kushi LH, Doyle C, McCullough M, Rock CL, Demark-Wahnefried W, et al. (2012) American Cancer Society Guidelines on nutrition and physical activity for cancer prevention: reducing the risk of cancer with healthy food choices and physical activity. CA Cancer J Clin 62(1): 30-67.

20. Beaber EF, Buist DS, Barlow WE, Malone KE, Reed SD, et al. (2014) Recent oral contraceptive use by formulation and breast cancer risk among women 20 to 49 years of age. Cancer Res 74(15): 4078-4089.

21. Manson JE, Chlebowski RT, Stefanick ML, Aragaki AK, Rossouw JE, et al. (2013) Menopausal hormone therapy and health outcomes during the intervention and extended poststopping phases of the Women's Health Initiative randomized trials. JAMA 310(13): 1353-1368.

22. Calle EE, Feigelson HS, Hildebrand JS, Teras LR, Thun MJ, et al. (2009) Postmenopausal hormone use and breast cancer associations differ by hormone regimen and histologic subtype. Cancer 115(5): 936-945.

23. Gail MH, Brinton LA, Byar DP, Corle DK, Green SB, et al. (1981) Projecting individualized probabilities of developing breast cancer for white females who are being examined annually. J Natl Cancer Inst 81(24): 1879-1886.

24. Zurrida S, Bassi F, Arnone P, Martella S, Del Castillo A, et al. (2011) The Changing Face of Mastectomy (from Mutilation to Aid to Breast Reconstruction). Int J Surg Oncol 2011: 980158.

25. Kummerow KL, Du L, Penson DF, Shyr Y, Hooks MA (2015) Nationwide trends in mastectomy for early-stage breast cancer. JAMA Surg 150(1): 9-16.

26. Rebbeck TR, Friebel T, Lynch HT, Neuhausen SL, van't Veer L, et al (2004) Bilateral prophylactic mastectomy reduces breast cancer risk in BRCA1 and BRCA2 mutation carriers: the PROSE Study Group. J Clin Oncol 22(6): 1055-1062.

27. Meijers-Heijboer H, van Geel B, van Putten WL, Henzen-Logmans SC Seynaeve C, et al. (2001) Breast cancer after prophylactic bilateral mastectomy in women with a BRCA1 or BRCA2 mutation. N Engl J Med 345(3): 159-164.

28. Davies KR, Brewster AM, Bedrosian I, Parker PA, Crosby MA, et al. (2016) Outcomes of contralateral prophylactic mastectomy in relation to familial history: a decision analysis (BRCR-D-16-00033). Breast Cancer Res 18(1): 93.

30. Fayanju OM, Stoll CR, Fowler S, Colditz GA, Margenthaler JA (2014) Contralateral prophylactic mastectomy after unilateral breast cancer: a systematic review and meta-analysis. Ann Surg 260(6): 1000-1010.

31. Fisher B, Anderson S, Bryant J, Margolese RG, Deutsch M, et al. (2002) Twenty-year follow-up of a randomized trial comparing tota mastectomy, lumpectomy, and lumpectomy plus irradiation for the treatment of invasive breast cancer. N Engl J Med 347(16): 1233-1241.

32. Early Breast Cancer Trialists' Collaborative Group (1995) Effects of radiotherapy and surgery in early breast cancer. An overview of the randomized trials. Early Breast Cancer Trialists' Collaborative Group. N Engl J Med 333(22): 1444-1455.

33. Hartmann LC, Schaid DJ, Woods JE, Crotty TP, Myers JL, et al. (1999) Efficacy of bilateral prophylactic mastectomy in women with a family history of breast cancer. N Engl J Med 340(2): 77- 84

33. Bedrosian I, Hu CY, Chang GJ (2010) Population-based study of contralateral prophylactic mastectomy and survival outcomes of breast cancer patients. J Natl Cancer Inst 102(6): 401-409.
34. van Verschuer VM, Maijers MC, van Deurzen CH, Koppert LB (2015) Oncological safety of prophylactic breast surgery: skin-sparing and nipple-sparing versus total mastectomy. Gland Surg 4(6): 467-475.

35. Hicken N (1940) Mastectomy: A clinical pathologic study demonstrating why most mastectomies result in incomplete removal of the mammary gland. Archives of Surgery 40(1): 6-14.

36. Griepsma M, de Roy van Zuidewijn DB, Grond AJ, Siesling S, et al (2014) Residual breast tissue after mastectomy: how often and where is it located? Ann Surg Oncol 21(4): 1260-1266.

37. Domchek SM, Friebel TM, Singer CF, Evans DG, Lynch HT, et al. (2010) Association of risk-reducing surgery in BRCA1 or BRCA2 mutation carriers with cancer risk and mortality. JAMA 304(9): 967-975.

38. Gahm J, Jurell G, Edsander-Nord A, Wickman M (2010) Patient satisfaction with aesthetic outcome after bilateral prophylactic mastectomy and immediate reconstruction with implants. J Plast Reconstr Aesthet Surg 63(2): 332-338.

39. Kurian AW, Canchola AJ, Gomez SL, Clarke CA (2016) Equivalent survival after nipple-sparing compared to non-nipple-sparing mastectomy: data from California, 1988-2013. Breast Cancer Res Treat $160(2): 333-338$

40. Howes BH, Watson DI, Xu C, Fosh B, Canepa M (2016) Quality of life following total mastectomy with and without reconstruction versus breast-conserving surgery for breast cancer: A case-controlled cohort study. J Plast Reconstr Aesthet Surg 69(9): 1184-1191.

41. Olsen MA, Nickel KB, Fox IK, Margenthaler JA, Ball KE, et al. (2015) Incidence of Surgical Site Infection Following Mastectomy With and Without Immediate Reconstruction Using Private Insurer Claims Data. Infect Control Hosp Epidemiol 36(8): 907-914.

42. Major M, Devulapalli C, Bello RJ, Baltodano PA, Reinhardt ME, et al. (2016) The Effect of Timing on Breast Reconstruction Outcomes in Diabetic Women. Plast Reconstr Surg Glob Open 4(10): e1090.

43. Howell A, Anderson AS, Clarke RB, Duffy SW, Evans DG, et al. (2014) Risk determination and prevention of breast cancer. Breast Cancer Res 16(5): 446.

44. Fisher B, Costantino JP, Wickerham DL, Redmond CK, Kavanah M, et al. (1998) Tamoxifen for prevention of breast cancer: report of the National Surgical Adjuvant Breast and Bowel Project P-1 Study. J Natl Cancer Inst 90(18): 1371-1388.

45. Khalkhali-Ellis Z, Christian AL, Kirschmann DA, Edwards EM, RezaieThompson M, et al. (2004) Regulating the tumor suppressor gene maspin in breast cancer cells: a potential mechanism for the anticancer properties of tamoxifen. Clin Cancer Res 10(2): 449-454.

46. Vogel VG, Costantino JP, Wickerham DL, Cronin WM (2003) National surgical adjuvant breast and bowel project update: prevention trials and endocrine therapy of ductal carcinoma in situ. Clin Cancer Res 9(1 Pt 2): 495S-501S

47. Madlensky L, Natarajan L, Tchu S, Pu M, Mortimer J, et al. (2011) Tamoxifen metabolite concentrations, CYP2D6 genotype, and breast cancer outcomes. Clin Pharmacol Ther 89(5): 718-725.

48. Kiyotani K, Mushiroda T, Imamura CK, Hosono N, Tsunoda T, et al (2010) Significant effect of polymorphisms in CYP2D6 and ABCC2 on clinical outcomes of adjuvant tamoxifen therapy for breast cancer patients. J Clin Oncol 28(8): 1287-1293.

49. Regan MM, Leyland-Jones B, Bouzyk M, Pagani O, Tang W, et al. (2012) CYP2D6 genotype and tamoxifen response in postmenopausal women with endocrine-responsive breast cancer: the breast international group 1-98 trial. J Natl Cancer Inst 104(6): 441-451.

50. Dickler MN, Norton L (2001) The MORE trial: multiple outcomes for raloxifene evaluation--breast cancer as a secondary end point: implications for prevention. Ann N Y Acad Sci 949: 134-142. 
51. Vogel VG (2009) The NSABP Study of Tamoxifen and Raloxifene (STAR) trial. Expert Rev Anticancer Ther 9(1): 51-60.

52. Goss PE, Ingle JN, Ales-Martinez JE, Cheung AM, Chlebowski RT, et al (2011) Exemestane for breast-cancer prevention in postmenopausal women. N Engl J Med 364(25): 2381-2391.

53. Chumsri S, Howes T, Bao T, Sabnis G, Brodie A (2011) Aromatase, aromatase inhibitors, and breast cancer. J Steroid Biochem Mol Biol 125(1-2):13-22.

54. (2016) Aromasin (exemestane) tablets package insert. New York NPIJ, USA.

55. Cuzick J, Sestak I, Baum M, Buzdar A, Howell A, et al. (2010) Effect of anastrozole and tamoxifen as adjuvant treatment for early-stage breast cancer: 10-year analysis of the ATAC trial. Lancet Oncol 11(12): $1135-1141$

56. Cuzick J, Sestak I, Forbes JF, Dowsett M, Knox J, et al. (2014) Anastrozole for prevention of breast cancer in high-risk postmenopausal women (IBIS-II): an international, double-blind, randomised placebocontrolled trial. Lancet 383(9922): 1041-1048.
57. Zendejas B, Moriarty JP, O’Byrne J, Degnim AC, Farley DR, et al. (2011) Cost-effectiveness of contralateral prophylactic mastectomy versus routine surveillance in patients with unilateral breast cancer. J Clin Oncol 29(22): 2993-3000.

58. Deshmukh AA, Cantor SB, Crosby MA, Dong W, Shen Y, et al. (2014) Cost of contralateral prophylactic mastectomy. Ann Surg Oncol 21(9): 2823-2830.

59. (2017) Anastrozole, Lexicomp Online, Hudson, Ohio: Lexi-Comp, Inc.; March 1, 2017.

60. (2016) Exemestane, Lexicomp Online, Hudson, Ohio: Lexi-Comp, Inc.; December 9, 2016.

61. Edwards BL, Hu Y, Stukenborg GJ et al. (2015) Cost-effectiveness of bilateral prophylactic mastectomy in patients at high risk for breast cancer without known BRCA mutation. American Society of Breast Surgeons Annual Meeting Presented, USA.

62. Bedrosian I, Yao K (2015) Contralateral Prophylactic Mastectomy: Anxiety, Knowledge and Shared Decision Making. Ann Surg Oncol 22(12): 3767-3768.

Your next submission with Juniper Publishers will reach you the below assets

- Quality Editorial service

- Swift Peer Review

- Reprints availability

- E-prints Service

- Manuscript Podcast for convenient understanding

- Global attainment for your research

- Manuscript accessibility in different formats

( Pdf, E-pub, Full Text, Audio)

- Unceasing customer service

Track the below URL for one-step submission https://juniperpublishers.com/online-submission.php 